\title{
A microlocal version of Cartan-Grauert's theorem
}

\author{
by I. V. Maresin and A. G. Sergeev (Moscow)
}

\begin{abstract}
Tuboids are tube-like domains which have a totally real edge and look asymptotically near the edge as a local tube over a convex cone. For such domains we state an analogue of Cartan's theorem on the holomorphic convexity of totally real domains in $\mathbb{R}^{n} \subset \mathbb{C}^{n}$.
\end{abstract}

1. Classical Cartan theorem. In his paper [3] H. Cartan proved the following theorem.

Theorem (Cartan). Let $\omega$ be a domain in $\mathbb{R}^{n}=\mathbb{R}^{n}+i 0 \subset \mathbb{C}^{n}$. Then $\omega$ has a fundamental system of neighborhoods $\Omega$ in $\mathbb{C}^{n}$ which are domains of holomorphy. In other words, for any domain $\Omega^{\prime}$ in $\mathbb{C}^{n}$, containing $\omega$, there exists a domain of holomorphy $\Omega$ such that $\omega \subset \Omega \subset \Omega^{\prime}$.

H. Grauert in [4] extended this theorem to domains lying on analytic totally real submanifolds in $\mathbb{C}^{n}$.

One way to prove Cartan's theorem is to construct the desired domain $\Omega$ as (the interior of) the intersection of a family of complex hyperboloids.

In more detail, denote by $\Omega_{x}^{\prime}$ the section of $\Omega^{\prime}$ by the "imaginary" plane $x+i \mathbb{R}^{n}$ through $x \in \Omega^{\prime} \cup \mathbb{R}^{n}=: \omega^{\prime}$ :

$$
\Omega_{x}^{\prime}=\left\{z=x+i y \in \mathbb{C}^{n}: z \in \Omega^{\prime}\right\} .
$$

Denote by $r(x)$ the "radius" of this section, i.e. $r(x)=\operatorname{dist}\left(x, \partial \Omega_{x}^{\prime}\right)$ for $x \in \omega^{\prime}$ and 0 otherwise. It is a lower semicontinuous function on $\mathbb{R}^{n}$.

A complex hyperboloid or a pseudoball is a domain of holomorphy in $\mathbb{C}^{n}$ of the form

$$
U\left(z_{0}, r\right)=\left\{z \in \mathbb{C}^{n}: \operatorname{Re}\left(z-z_{0}\right)^{2}+r^{2}>0\right\}
$$

1991 Mathematics Subject Classification: Primary 32A07.

Key words and phrases: totally real submanifolds, domains of holomorphy, tuboids.

Research partially supported by the Russian Foundation for Basic Research (grants 98-01-00640 and 96-15-96131). 
The section $U_{x}\left(z_{0}, r\right)$ of the hyperboloid $U\left(z_{0}, r\right)$ by the "imaginary" plane $x+i \mathbb{R}^{n}$ through $x \in \mathbb{R}^{n}$ is the ball of radius $\sqrt{\left(x-x_{0}\right)^{2}+r^{2}}$ with centre at $y_{0}$.

Now we define the domain of holomorphy $\Omega$ as the intersection of all complex hyperboloids with centres on $\omega^{\prime}$ :

$$
\Omega=\operatorname{Int} \bigcap_{x \in \omega^{\prime}} U(x, r(x))
$$

where Int denotes interior.

By construction, $\Omega \subset \Omega^{\prime}$. We show that $\Omega \supset \omega^{\prime} \supset \omega$. For any $x_{0} \in \omega^{\prime}$ we consider a neighborhood $o\left(x_{0}\right)=\left\{x \in \omega^{\prime}:\left|x-x_{0}\right|<d_{0}\right\}$ with compact closure in $\omega^{\prime}$. Set $r_{0}=\inf \left\{r(x): x \in \overline{o\left(x_{0}\right)}\right\}$ (this is positive due to the semicontinuity of $r(x))$. We assert that $\Omega$ contains a neighborhood of $x_{0}$ of the form

$$
O\left(x_{0}\right)=\left\{z=x+i y:\left|x-x_{0}\right|<\varepsilon,|y|<\varepsilon\right\},
$$

where $\varepsilon=\frac{1}{2} \min \left\{d_{0}, r_{0}\right\}$. Indeed, if the centre $x^{\prime}$ of a hyperboloid $U\left(x^{\prime}, r\left(x^{\prime}\right)\right)$ is "close" to $x_{0}$, namely, if $x^{\prime} \in o\left(x_{0}\right)$, then its section $U_{x}\left(x^{\prime}, r^{\prime}\right)$ at any point $x$ with $\left|x-x_{0}\right|<d_{0}$ is the ball of radius $r^{\prime} \geq r\left(x^{\prime}\right) \geq r_{0}$ with centre at $y_{0}=0$, containing $O_{x}\left(x_{0}\right)$. If the distance from $x^{\prime}$ to $x_{0}$ is greater than $d_{0}$, then the radius of $U_{x}\left(x^{\prime}, r^{\prime}\right)$ at any point $x$ with $\left|x-x_{0}\right|<d_{0} / 2$ satisfies $r^{\prime} \geq\left|x-x^{\prime}\right| \geq d_{0} / 2 \geq \varepsilon$ and the section $U_{x}\left(x^{\prime}, r\left(x^{\prime}\right)\right)$ again contains $O_{x}\left(x_{0}\right)$.

This argument shows that $\Omega$ is open and contains $\omega^{\prime} \supset \omega$. Moreover, it is connected because $\omega^{\prime}$ is connected and the sections $\Omega_{x}$ are convex. Finally, $\Omega$ is a domain of holomorphy, being the interior of the intersection of complex hyperboloids.

2. Tuboids. Recall that a local tube over a domain $\omega \subset \mathbb{R}^{n}$ with profile $V$ is a domain in $\mathbb{C}^{n}$ of the form

$$
T_{r}(\omega, V)=\omega+i V_{r}=\left\{z=x+i y \in \mathbb{C}^{n}: x \in \omega, y \in V_{r}\right\}
$$

where $V$ is an open connected (non-empty) cone in $\mathbb{R}^{n}$, and $V_{r}:=V \cap$ $\left\{y \in \mathbb{R}^{n}:|y|<r\right\}$.

Tuboids are domains in $\mathbb{C}^{n}$ which look like local tubes with profile varying from point to point in $\mathbb{R}^{n}$. First we define more precisely what we mean by the varying profile.

Definition 1. Let $\omega$ be a domain in $\mathbb{R}^{n}$. A profile over $\omega$ is a domain $\Lambda$ in $\mathbb{C}^{n}$ of the form

$$
\Lambda=\Lambda(\omega)=\left\{z=x+i y: x \in \omega, y \in \Lambda_{x}\right\}
$$

where $\Lambda_{x}$ is an open connected (non-empty) cone in $\mathbb{R}^{n}$.

The set

$$
\dot{\Lambda}=\left\{z=x+i y: x \in \omega, y \in \dot{\Lambda}_{x}\right\}
$$


where $\dot{\Lambda}_{x}$ is the intersection of $\Lambda_{x}$ with the unit sphere $S^{n-1}$ in $\mathbb{R}^{n}$, will be called the base of the profile $\Lambda(\omega)$. (We always assume that the equality $\dot{\Lambda}_{x}=S^{n-1}$ for some $x \in \mathbb{R}^{n}$ implies $\Lambda_{x}=\mathbb{R}^{n}$.) A profile $\Lambda=\Lambda(\omega)$ is compactly supported in a profile $\Lambda^{\prime}=\Lambda^{\prime}(\omega), \Lambda \Subset \Lambda^{\prime}$, if the closure of the base $\dot{\Lambda}$ is contained in the base $\dot{\Lambda}^{\prime}$.

For a profile $\Lambda=\Lambda(\omega)$ we define its fiberwise convex hull as a profile over $\omega$ of the form

$$
\operatorname{ch} \Lambda=\left\{z=x+i y: x \in \omega, y \in \operatorname{ch} \Lambda_{x}\right\}
$$

where $\operatorname{ch} \Lambda_{x}$ is the convex hull of the cone $\Lambda_{x}$. A profile is called fiberwise convex iff $\operatorname{ch} \Lambda=\Lambda$. Accordingly, the base $\dot{\Lambda}$ of a profile $\Lambda$ is called fiberwise convex if $\Lambda$ is fiberwise convex.

Definition 2. A tuboid in $\mathbb{C}^{n}$ with profile $\Lambda=\Lambda(\omega)$ is a domain $\Omega$ in $\mathbb{C}^{n}$ of the form

$$
\Omega=\Omega(\Lambda)=\left\{z=x+i y: x \in \omega, y \in \Omega_{x}\right\},
$$

where $\Omega_{x}$ is a domain in $\mathbb{R}^{n}$, such that the following condition is satisfied: for any $x \in \omega$ and any open connected cones $V^{\prime}, V^{\prime \prime}$ in $\mathbb{R}^{n}$ such that $V^{\prime} \Subset$ $\Lambda_{x} \Subset V^{\prime \prime}$ there exist a neighborhood $O(x)$ of $x$ in $\mathbb{C}^{n}$ and a number $r>0$ such that

$$
T_{r}\left(o(x), V^{\prime}\right) \subset \Omega \cap O(x) \subset T_{r}\left(o(x), V^{\prime \prime}\right)
$$

with $o(x):=O(x) \cap \mathbb{R}^{n}$. In other words, the intersection of $\Omega$ with a sufficiently small neighborhood $O(x)$ must, on the one hand, contain some local tube with profile $V^{\prime}$ and, on the other hand, be contained in some local tube with profile $V^{\prime \prime}$.

One can imagine a tuboid with profile $\Lambda(\omega)$ as a domain with the edge $\omega$ which approximates the cone $\Lambda_{x}$ "asymptotically" near each $x \in \omega$. Local tubes and profiles can themselves serve as examples of tuboids. The notion of tuboid was introduced by J. Bros and D. Iagolnitzer in [1, 2].

The definitions of the profile and tuboid are easily extended to the case where the edge $\mathbb{R}^{n}$ is replaced by a totally real submanifold in $\mathbb{C}^{n}$. Namely, let $M$ be a smooth totally real submanifold of dimension $n$ in $\mathbb{C}^{n}=\mathbb{R}_{(x)}^{n}+i \mathbb{R}_{(y)}^{n}$ and let $\omega$ be a domain on $M$. Suppose that for any $z \in \omega$ the "imaginary" plane $z+i \mathbb{R}_{(y)}^{n}$ is transversal to $M$ at $z$. Denote by $\left\{\Lambda_{z}\right\}$ a family of open connected (non-empty) cones in $\mathbb{R}_{(y)}^{n}$ parametrized by points $z \in \omega$. A profile over $\omega$ is a domain $\Lambda$ in $\mathbb{C}^{n}$ of the form

$$
\Lambda=\bigcup_{z \in \omega}\left\{z+\Lambda_{z}\right\} \text {. }
$$

A profile $\Lambda$ will be called a tube profile if for any $z \in \omega$ the cone $\Lambda_{z}$ equals $\Lambda_{0}$, i.e. does not depend on $z$. Replacing the cone $\Lambda_{0}$ in this definition by its intersection with the ball $B(0, r)$ of radius $r$ with centre at the origin, we 
get the definition of a local tube profile. A tuboid over $\omega$ is a domain of the form

$$
\Omega=\bigcup_{z \in \omega}\left\{z+\Omega_{z}\right\}
$$

where $\Omega_{z}$ is a domain in $\mathbb{R}_{(y)}^{n}$ which can be approximated at points of $\omega$ by local tube profiles in the same sense as in Definition 2 above.

3. Cartan-Grauert's theorem for tuboids. We now formulate a generalization of Cartan-Grauert's theorem to tuboids.

The standard complex metric $(z, z)_{\mathbb{C}}=z_{1}^{2}+\ldots+z_{n}^{2}$ on $\mathbb{C}^{n}$ induces a real metric on $\mathbb{C}^{n}$, identified with $\mathbb{R}^{2 n}$, of signature $(n, n)$ given by

$$
(z, z)_{\mathbb{R}}:=\operatorname{Re}(z, z)=(x, x)-(y, y) \quad \text { for } z=x+i y \in \mathbb{C}^{n} .
$$

Denote by $E$ the vector space $\mathbb{R}^{2 n}$ provided with this metric. A vector $z \in E$ is real-like if $(z, z)_{\mathbb{R}}>0$, imaginary-like if $(z, z)_{\mathbb{R}}<0$, and null if $(z, z)_{\mathbb{R}}=0$. Accordingly, a linear subspace in $E$ is called real-like (resp. imaginary-like, null) if every non-zero vector in this subspace is real-like (resp. imaginarylike, null).

We say that a smooth submanifold $M$ in $E$ is real-like if the tangent space $T_{z_{0}} M$ at any point $z_{0} \in M$ is real-like. Evidently, such a submanifold is always totally real. A submanifold $M$ is strictly real-like if it coincides with the graph of a smooth map

$$
F: \mathbb{R}_{(x)}^{n} \rightarrow \mathbb{R}_{(y)}^{n}
$$

with derivative satisfying the condition

$$
\left\|F^{\prime}(x)\right\|<1 \quad \text { for any } x \in \mathbb{R}_{(x)}^{n} .
$$

THEOREM. Let $M$ be a $C^{2}$-smooth strictly real-like submanifold in $E \cong \mathbb{C}^{n}$, and $\omega$ be a domain on $M$. Suppose that $\Lambda$ is a fiberwise convex profile over $\omega$. Then for any tuboid $\Omega^{\prime}$ with profile $\Lambda$ there exists a tuboid $\Omega \subset \Omega^{\prime}$ with the same profile which is a domain of holomorphy in $\mathbb{C}^{n}$.

In order to obtain the classical Cartan theorem of Section 1 from the above theorem, take $M=\mathbb{R}_{(x)}^{n}, \Lambda=\mathbb{R}^{n}$. Our theorem may be considered as a "microlocal" variant of Cartan-Grauert's theorem because replacing the full profile $\Lambda=\mathbb{R}^{n}$ in the classical case by an arbitrary profile $\Lambda$ corresponds to localization in "conormal directions". An extension of Cartan's theorem to tuboids was proved by J. Bros and D. Iagolnitzer in $[1,2]$ in the case when $M$ is $\mathbb{R}^{n}$ or real-analytic.

The condition of fiberwise convexity of the profile $\Lambda$ is not very essential since for any tuboid $\Omega^{\prime}$ over $\omega$ with profile $\Lambda$ we can find a tuboid $\Omega^{\prime \prime}$ over $\omega$ with profile $\operatorname{ch} \Lambda$ such that any function holomorphic in $\Omega$ extends holomorphically to $\Omega^{\prime \prime}$. This is a microlocal version of Bochner's tube theorem. 
It was proved for tuboids (in the case when $M$ is $\mathbb{R}^{n}$ or real-analytic) in $[1,2]$.

We give here a sketch of the proof of the above theorem; the details are given in [5].

We shall construct the desired tuboid $\Omega$ using the same idea as in the proof of the classical Cartan theorem, as the intersection of a family of complex pseudoballs in $E$.

At every point $\xi \in M$ we have a decomposition of the tangent space

$$
T_{\xi} E \cong E=\mathcal{R}_{\xi} \oplus \mathcal{J}_{\xi},
$$

where $\mathcal{R}_{\xi}=T_{\xi} M$ and $\mathcal{J}_{\xi}$ is its orthogonal complement in $E$. We introduce a curvature function of $M$ determined by the norm of the second derivative $\left\|F^{\prime \prime}(\xi)\right\|$ at $\xi \in M$ (assuming that $F(\xi)=F^{\prime}(\xi)=0$ ). Having the curvature function one can define a "characteristic radius" $r(\xi)>0$ of $M$ at $\xi$ which is a continuous function on $M$. We denote by $B_{\xi}$ the ball of radius $r(\xi)$ in $\mathcal{J}_{\xi} \subset \xi+E$. The union of the balls $B_{\xi}$ over all $\xi \in M$ forms a tube neighborhood $B$ of $M$ in $E$ which can also be identified with the ball normal bundle of $M$ in $E$. Points in $B$ have "spherical coordinates" $(\xi, s, r)$ where $\xi \in M, s$ is a point of the unit sphere in $\mathcal{J}_{\xi}, 0 \leq r<r(\xi)$.

The desired tuboid $\Omega$ is constructed in several steps by "cutting off superfluous parts" from the original tuboid $\Omega^{\prime}$. We recall that $\Omega^{\prime}$ has the fiberwise convex profile $\Lambda$. The first step is to construct a tuboid $\Omega_{1} \subset B$ over $\omega$ with the same profile but having convex spherical sections. In terms of the spherical coordinates on $B$, introduced above, this means that all sections $\Omega_{1, \xi, r}$ of $\Omega_{1}$ with fixed $(\xi, r)$ are convex (i.e. the cones generated by $\Omega_{1, \xi, r}$ are convex). Moreover, $\Omega_{1} \subset \Omega$.

The next step is to construct a tuboid $\Omega_{2} \subset \Omega_{1}$ over $\omega$ with the same profile having fiberwise spherically convex fibres.

We recall the definition of spherical convexity. Any bounded convex domain $D$ in $\mathbb{R}^{n}$, contained, for instance, in the ball of radius $R$, may be represented as the intersection of support hyperplanes containing $D$. Let $y \in \partial D$. Replace the hyperplane supporting $D$ at $y$ by the ball $B_{y}$ of radius $R$ which is tangent to the boundary of the support hyperplane at $y$. Taking the intersection of all such balls $B_{y}$ over all $y \in \partial D$ we obtain a set

$$
\breve{D}=\bigcap_{y \in \partial D} B_{y}
$$

which is called the spherical polar of $D$. By construction, $\breve{D} \subset D$. The domain $D$ is called spherically convex iff $\breve{D}=D$.

At the second step we construct a tuboid $\Omega_{2} \subset \Omega_{1} \subset B$ whose fibres $\Omega_{2, \xi}$ are spherically convex with respect to the ball $B_{\xi}$. We denote by $\breve{\Omega}_{2}$ the 
spherical polar of $\Omega_{2}$, i.e. the set formed by the fiberwise spherical polars $\Omega_{2, \xi}$ of $\Omega_{2, \xi}$ with respect to the balls $B_{\xi}$ for $\xi \in \omega$.

The last step is the construction of the tuboid $\Omega \subset \Omega_{2}$ by taking the interior of the intersection of all pseudoballs $U((\xi, s, r), r(\xi))$ with centres at points $(\xi, s, r) \in \breve{\Omega}_{2}$ of "characterictic radius" $r(\xi)$ for $\xi \in \omega$. Then $\Omega \subset \Omega^{\prime}$ is a tuboid over $\omega$ with the same profile $\Lambda$ which is a domain of holomorphy.

\section{References}

[1] J. Bros and D. Iagolnitzer, Tuboïdes et structure analytique des distributions, Sém. Goulaouic-Lions-Schwartz, nos. 16, 18, 1975.

[2] -, 一, Tuboïdes dans $\mathbb{C}^{n}$ et généralisation d'un théorème de Grauert, Ann. Inst. Fourier (Grenoble) 26 (1976), no. 3, 49-72.

[3] H. Cartan, Variétés analytiques réelles et variétés analytiques complexes, Bull. Soc. Math. France 85 (1957), 77-100.

[4] H. Grauert, On Levi's problem and the embedding of real analytic manifolds, Ann. of Math. (2) 68 (1958), 460-472.

[5] I. V. Maresin, Cartan-Grauert's theorem for tuboids with a curvilinear edge, Math. Notes 64 (1998), no. 6.

Steklov Mathematical Institute

Gubkina 8, 117966, GSP-1

Moscow, Russia

E-mail: q@maresin.mccme.rssi.ru sergeev@genesis.mi.ras.ru 